International Journal of Modern Physics B

(C) World Scientific Publishing Company

\title{
EXACT RESULTS OF THE MIXED-SPIN ISING MODEL ON A DECORATED SQUARE LATTICE WITH TWO DIFFERENT DECORATING SPINS OF INTEGER MAGNITUDES
}

\author{
LUCIA ČANOVÁ, JOZEF STREČKA, and MICHAL JAŠČUR \\ Department of Theoretical Physics and Astrophysics, Faculty of Science, \\ P. J. Šafárik University, Park Angelinum 9, 04001 Košice, Slovak Republic \\ lucia.canova@pobox.sk
}

Received Day Month Year

Revised Day Month Year

\begin{abstract}
The mixed-spin Ising model on a decorated square lattice with two different decorating spins of the integer magnitudes $S_{\mathrm{B}}=1$ and $S_{\mathrm{C}}=2$ placed on horizontal and vertical bonds of the lattice, respectively, is examined within an exact analytical approach based on the generalized decoration-iteration mapping transformation. Besides the groundstate analysis, finite-temperature properties of the system are also investigated in detail. The most interesting numerical result to emerge from our study relates to a striking critical behaviour of the spontaneously ordered 'quasi-1D' spin system. It was found that this quite remarkable spontaneous order arises when one sub-lattice of the decorating spins (either $S_{\mathrm{B}}$ or $S_{\mathrm{C}}$ ) tends towards their 'non-magnetic' spin state $S=0$ and the system becomes disordered only upon further single-ion anisotropy strengthening. The effect of single-ion anisotropy upon the temperature dependence of the total and sublattice magnetization is also particularly investigated.
\end{abstract}

Keywords: Ising model; phase transitions; exact results.

\section{Introduction}

Over the last six decades, a considerable research interest has been devoted to determine a critical behaviour and other statistical properties of various latticestatistical models, which enable a deeper understanding of order-disorder phenomena in magnetic solids. In this respect, the planar Ising model takes a prominent place, since it represents perhaps the simplest lattice-statistical model for which a complete exact solution is known since the Onsager's pioneering work 1 . Exactly soluble planar Ising models ${ }^{2}$ provide a convincing evidence for many controversial results predicted in the phase transition theory. Besides, these models also provide a good testing ground for many approximative theories. It should be stressed, nevertheless, that a precise treatment of two-dimensional (2D) Ising models is often connected with considerable difficulties, which relate to the usage of sophisticated mathematical methods when applying them to more complex models describing, for instance, spin systems with interactions beyond nearest neighbours ${ }^{3}$, frus- 
trated spin systems 4 , or higher-spin models with (or without) single-ion anisotropy and biquadratic interactions 5 . Up to now, exact results for Ising models on the square ${ }^{1}$, triangular and honeycomb 6 , kagomé $e^{7}$, extended kagomé ${ }^{8}$, bathroom-tile 9, orthogonal-dimer 10 and ruby 11 lattice, as well as on various irregular $2 \mathrm{D}$ lattices, such as union jack (centered square) $\frac{12}{12}$, pentagona 13 , square-kagomé ${ }^{14}$, or two topologically distinct square-hexagona 15 lattices, have been obtained.

The Ising systems consisting of mixed spins of different magnitudes, which are usually called also as mixed-spin Ising models, belong among the most interesting extensions of the standard spin-1/2 Ising model. These models have recently enjoyed a great scientific interest predominantly due to their rich critical behaviour they display. Another aspect, which started to attract a scientific interest to the mixedspin Ising models, relates to a theoretical modeling of magnetic structures suitable for describing a ferrimagnetism of a certain class of insulating magnetic materials. In this respect, the ferrimagnetic mixed-spin Ising models are very interesting also from the experimental point of view.

Despite a significant amount of effort, there are known only few examples of exactly solvable mixed-spin Ising models, yet. Using the generalized forms of decoration-iteration and star-triangle mapping transformations, the mixed spin$1 / 2$ and spin- $S(S \geq 1)$ Ising models on the honeycomb, diced and several decorated planar lattices were exactly examined long years agd 16 . Later on, both the aforementioned transformation procedures were generalized in order to account for the single-ion anisotropy effect. In this way generalized mapping transformations were employed to exactly investigate the influence of uniaxial and biaxial singleion anisotropies on magnetic properties of the mixed-spin Ising systems on the honeycomb 17 , bathroom-tile ${ }^{18}$ or diced lattice ${ }^{19}$ and several decorated planar lattices 20|21. To the best of our knowledge, these are the only mixed-spin planar Ising models with generally known exact solutions with exception to several mixed-spin Ising models on the Bethe (Cayley tree) lattices studied within an approach based on exact recursion equations 22 . Among the remarkable models, for which a precise solution is restricted to a certain subspace of interaction parameters only, one should also mention the mixed-spin Ising model on the union jack lattice treated within the mapping onto a symmetric (zero-field) eight-vertex mode 23.

Exactly solvable mixed-spin Ising models on 2D lattices, the bonds of which are decorated in various fashion by additional spins, are therefore of particular research interest (see Ref. $\frac{24}{}$ and references cited therein). Among the systems, which belong to the class of exactly solved decorated Ising models, one could mention, at least, the original ferrimagnetic model introduced by Syozi and Nakand 25 , partly 26 and multiply 27 decorated models showing reentrant phase transitions, ANNNI models 28 , diluted models of ferromagnetism 29 , decorated model systems with classical $\nu$ dimensional vector spins 30 , Fisher's super-exchange model and its other variants 31 , as well as the models with higher decorating spins 16 . It is worth mentioning that exact solutions of these spin model systems have furnished answers to questions 
interesting from the academic point of view (scaling and universality hypothesis, reentrant phase transitions) as well as from the experimental viewpoint (dilution, technological application of ferrimagnets). The great potential of ferrimagnets with respect to technological applications has also stimulated exploration of the singleion anisotropy effect upon ferrimagnetic features of the mixed spin- $1 / 2$ and spin- $S$ Ising models on the wholly 20 and partly 21 decorated lattices.

Recently, Kaneyosh $\sqrt{32}$ has proposed another notable example of the decorated Ising model on a square lattice, the horizontal and vertical bonds of which are occupied by decorating spins of different magnitudes. Up to now, this model system has been examined by the use of the approach based on the differential operator technique 33 , whereas an accuracy of obtained results determined the Bethe-PeierlsWeiss approximation used for the undecorated lattice 32 . The purpose of this work is therefore to extend the class of exactly solvable Ising models by providing an accurate solution for the mixed-spin Ising model on the square lattice with decorating spins of two different magnitudes $S_{\mathrm{B}}$ and $S_{\mathrm{C}}$. Exact results for the model system under consideration are obtained by the use of the generalized decoration-iteration transformation 24 constituting an exact correspondence with an effective spin- $1 / 2$ Ising model on the anisotropic square (rectangular) lattice, the exact solution of which is well known 1 . Within the framework of the used mapping method, we will focus our attention first of all on the influence of the single-ion anisotropy on the critical behaviour and phase diagrams. Besides, temperature dependence of the total and sub-lattice magnetization will be also particularly examined.

The outline of this paper is as follows. In Section 2, the detailed description of the model system will be presented and then, some basic aspects of the used transformation method will be also shown. Section 3 deals with the interpretation of the most interesting numerical results for the mixed-spin model system with decorating spins of two different integer magnitudes $S_{\mathrm{B}}=1$ and $S_{\mathrm{C}}=2$. Finally, some concluding remarks are mentioned in Section 4

\section{Model and method}

We consider the mixed-spin Ising model on a decorated square lattice with decorating spins of two different magnitudes $S_{\mathrm{B}}$ and $S_{\mathrm{C}}$ placed on its horizontal and vertical bonds, respectively, as is schematically illustrated in Fig. 1 In this figure, the vertex sites of the original square lattice labeled by white circles are occupied by the spin- $S_{\mathrm{A}}$ atoms, while the decorating sites denoted by black and hatched circles are occupied by the spin- $S_{\mathrm{B}}$ and spin- $S_{\mathrm{C}}$ atoms, respectively. The total Hamiltonian of the model system defined upon the underlying square lattice reads

$$
\mathcal{H}=J_{\mathrm{AB}} \sum_{(i, m)}^{2 N} S_{i}^{z} S_{m}^{z}+J_{\mathrm{AC}} \sum_{(i, n)}^{2 N} S_{i}^{z} S_{n}^{z}-D_{\mathrm{B}} \sum_{m \in \mathrm{B}}^{N}\left(S_{m}^{z}\right)^{2}-D_{\mathrm{C}} \sum_{n \in \mathrm{C}}^{N}\left(S_{n}^{z}\right)^{2},
$$

where $S_{i}^{z}= \pm 1 / 2, S_{m}^{z}=-S_{\mathrm{B}},-S_{\mathrm{B}}+1, \ldots, S_{\mathrm{B}}$ and $S_{n}^{z}=-S_{\mathrm{C}},-S_{\mathrm{C}}+1, \ldots, S_{\mathrm{C}}$ denote three different Ising spin variables of the spin- $S_{\mathrm{A}}$, spin- $S_{\mathrm{B}}$ and spin- $S_{\mathrm{C}}$ atoms 


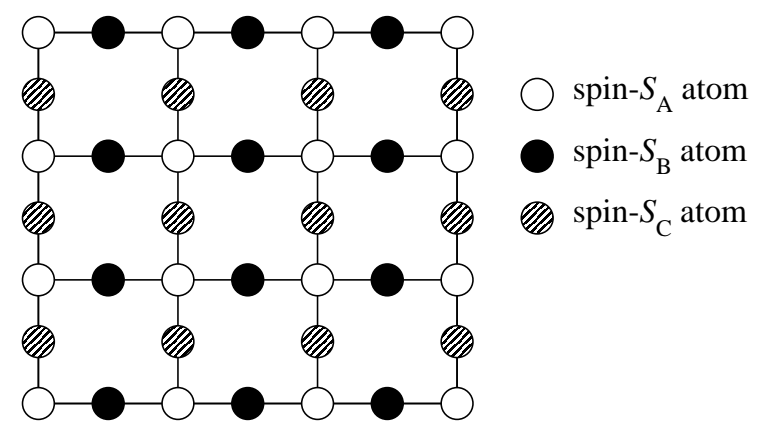

Fig. 1. Part of the mixed-spin Ising model on the decorated square lattice. The white circles denote spin- $1 / 2$ atoms, while the black and hatched circles denote the decorating spin- $S_{\mathrm{B}}$ and spin- $S_{\mathrm{C}}$ atoms, respectively.

located at $i$ th, $m$ th and $n$th lattice site, respectively. The first two summations are carried out over the nearest-neighbour A-B and A-C pairs, respectively, while the other two summations run over the decorating B and C lattice sites. Accordingly, the parameters $J_{\mathrm{AB}}$ and $J_{\mathrm{AC}}$ stand for exchange couplings between the nearest A-B and A-C neighbours, and the terms $D_{\mathrm{B}}$ and $D_{\mathrm{C}}$ measure a strength of the uniaxial single-ion anisotropy acting on the spin- $S_{\mathrm{B}}$ and spin- $S_{\mathrm{C}}$ atoms, respectively. Finally, $N$ denotes a total number of sites of the original square lattice.

The crucial step of our calculation represents the calculation of the partition function of the system. In view of further manipulations, it is useful to write the total Hamiltonian (1) in the form

$$
\mathcal{H}=\sum_{m=1}^{N} \mathcal{H}_{m}^{\mathrm{B}}+\sum_{n=1}^{N} \mathcal{H}_{n}^{\mathrm{C}},
$$

where the first (second) term represents the summation over bond Hamiltonians each involving all interaction terms associated with the spin- $S_{\mathrm{B}}\left(S_{\mathrm{C}}\right)$ atoms residing the $m$ th $(n$ th) decorating site of the lattice

$$
\mathcal{H}_{k}^{\mathrm{p}}=J_{\mathrm{Ap}} S_{k}^{z}\left(S_{i}^{z}+S_{i+1}^{z}\right)-D_{\mathrm{p}}\left(S_{k}^{z}\right)^{2},
$$

where $\mathrm{p}=\mathrm{B}$ or $\mathrm{C}$ and $k=m$ or $n$. The parameters $\mathrm{p}$ and $k$ in Eq. (3) specify, respectively, the decorating atom and its position in the lattice. Therefore, it is quite evident that if $k=m$, then one considers $\mathrm{p}=\mathrm{B}$ and if $k=n$, one sets $\mathrm{p}=\mathrm{C}$. The partition function of the considered mixed-spin system can be partially factorized into the product

$$
\mathcal{Z}=\sum_{\left\{S_{\mathrm{A}}\right\}} \prod_{m, n}^{N} \sum_{S_{m}^{z}=-S_{\mathrm{B}}}^{S_{\mathrm{B}}} \exp \left(-\beta \mathcal{H}_{m}^{\mathrm{B}}\right) \sum_{S_{n}^{z}=-S_{\mathrm{C}}}^{S_{\mathrm{C}}} \exp \left(-\beta \mathcal{H}_{n}^{\mathrm{C}}\right),
$$

where $\beta=1 /\left(k_{\mathrm{B}} T\right), k_{\mathrm{B}}$ is Boltzmann's constant and $T$ stands for the absolute temperature. The product in Eq. (4) is taken over all decorating lattice sites occupied 
by the spin- $S_{\mathrm{B}}$ and spin- $S_{\mathrm{C}}$ atoms, while the symbol $\sum_{\left\{S_{\mathrm{A}}\right\}}$ means the summation over all available spin configurations of the spin- $S_{\mathrm{A}}$ atoms. Finally, the next two summations are performed over all $\left(2 S_{\mathrm{B}}+1\right)$ and $\left(2 S_{\mathrm{C}}+1\right)$ possible spin states of the decorating spin- $S_{\mathrm{B}}$ and spin- $S_{\mathrm{C}}$ atoms, respectively. The structure of the latter relation immediately implies a possibility of applying the generalized decorationiteration mapping transformation $16 \mid 24$

$$
\sum_{S_{k}^{z}=-S_{\mathrm{p}}}^{S_{\mathrm{p}}} \exp \left(-\beta \mathcal{H}_{k}^{\mathrm{p}}\right)=A_{\mathrm{p}} \exp \left(\beta R_{\mathrm{p}} S_{i}^{z} S_{i+1}^{z}\right) .
$$

From the physical point of view, the mapping (5) removes all interaction parameters associated with one decorating spin $\left(S_{\mathrm{B}}\right.$ or $\left.S_{\mathrm{C}}\right)$ and replaces them by a new effective interaction $\left(R_{\mathrm{B}}\right.$ or $\left.R_{\mathrm{C}}\right)$ between the remaining vertex spins $S_{i}^{z}$ and $S_{i+1}^{z}$. Notice that a general validity of the mapping relation (5) necessitates a self-consistency condition to be satisfied, which means that it must hold independently of the spin states of both vertex spins $S_{i}^{z}$ and $S_{i+1}^{z}$. It can be readily proved that a direct substitution of four possible spin configurations of vertex spins $S_{i}^{z}$ and $S_{i+1}^{z}$ into the formula (5) gives just two independent equations, which unambiguously determine the mapping parameters $A_{\mathrm{p}}$ and $R_{\mathrm{p}}$

$$
A_{\mathrm{p}}=\left(W_{\mathrm{p} 1} W_{\mathrm{p} 2}\right)^{1 / 2}, \quad \beta R_{\mathrm{p}}=2 \ln \left(\frac{W_{\mathrm{p} 1}}{W_{\mathrm{p} 2}}\right)
$$

where the functions $W_{\mathrm{p} 1}$ and $W_{\mathrm{p} 2}$ are expressed as

$$
\begin{aligned}
& W_{\mathrm{p} 1}=\sum_{n=-S_{\mathrm{p}}}^{S_{\mathrm{p}}} \exp \left(\beta D_{\mathrm{p}} n^{2}\right) \cosh \left(\beta n J_{\mathrm{Ap}}\right), \\
& W_{\mathrm{p} 2}=\sum_{n=-S_{\mathrm{p}}}^{S_{\mathrm{p}}} \exp \left(\beta D_{\mathrm{p}} n^{2}\right) .
\end{aligned}
$$

After straightforward substitution of the transformation relation (5) into the formula (4), one easily obtains an exact relation between the partition function $\mathcal{Z}$ of the mixed-spin Ising model on the decorated square lattice and the partition function $\mathcal{Z}_{0}$ of the undecorated spin-1/2 Ising model on the corresponding rectangular lattice with two different nearest-neighbour couplings $R_{\mathrm{B}}$ and $R_{\mathrm{C}}$ in the horizontal and vertical directions, respectively,

$$
\mathcal{Z}\left(\beta, J_{\mathrm{AB}}, J_{\mathrm{AC}}, D_{\mathrm{B}}, D_{\mathrm{C}}\right)=A_{\mathrm{B}}^{N} A_{\mathrm{C}}^{N} \mathcal{Z}_{0}\left(\beta, R_{\mathrm{B}}, R_{\mathrm{C}}\right) .
$$

The mapping relation (9) represents the central result of our calculation, since it formally completes the exact solution of the partition function $\mathcal{Z}$ with regard to the known exact result for the partition function $\mathcal{Z}_{0}$ of the spin-1/2 Ising model on the rectangular lattice ${ }^{1}$. Actually, the equation (9) can be utilized for calculation of some important physical quantities, such as Gibbs free energy, internal energy, magnetization, correlation functions, specific heat, etc. Furthermore, by combining 
6 Lucia Čanová et al.

Eq. (9) with commonly used exact mapping theorems $\sqrt{34}$ and the differential operator technique 33 , one easily proves a validity of following exact relations for the sublattice magnetization $m_{\mathrm{A}}, m_{\mathrm{B}}$ and $m_{\mathrm{C}}$ coincided to the spin- $S_{\mathrm{A}}$, spin- $S_{\mathrm{B}}$, and spin$S_{\mathrm{C}}$ atom of the mixed-spin Ising model, respectively:

$$
\begin{aligned}
& m_{\mathrm{A}} \equiv\left\langle S_{i}^{z}\right\rangle=\left\langle S_{i}^{z}\right\rangle_{0}=m_{0}, \\
& m_{\mathrm{B}} \equiv\left\langle S_{m}^{z}\right\rangle=2 m_{\mathrm{A}} F_{\mathrm{B}}\left(J_{\mathrm{AB}}\right), \\
& m_{\mathrm{C}} \equiv\left\langle S_{n}^{z}\right\rangle=2 m_{\mathrm{A}} F_{\mathrm{C}}\left(J_{\mathrm{AC}}\right) .
\end{aligned}
$$

Above, the symbols $\langle\ldots\rangle$ and $\langle\ldots\rangle_{0}$ denote standard canonical averages performed over the mixed-spin Ising model defined by the Hamiltonian (1) and its corresponding spin-1/2 Ising model on the rectangular lattice, respectively. The functions $F_{\mathrm{p}}(x)$ $(\mathrm{p}=\mathrm{A}, \mathrm{B})$ stand for

$$
F_{\mathrm{p}}(x)=-\frac{\sum_{n=-S_{\mathrm{p}}}^{S_{\mathrm{p}}} n \exp \left(\beta D_{\mathrm{p}} n^{2}\right) \sinh (\beta n x)}{\sum_{n=-S_{\mathrm{p}}}^{S_{\mathrm{p}}} \exp \left(\beta D_{\mathrm{p}} n^{2}\right) \cosh (\beta n x)} .
$$

It should be pointed out that with regard to Eq. (10), the sub-lattice magnetization $m_{\mathrm{A}}$ directly equals to the corresponding spontaneous magnetization $m_{0}$ of the spin$1 / 2$ Ising model on the rectangular lattice with the effective horizontal and vertical coupling constants given by Eq. (6). The exact result for the magnetization of this model system is known for several years 35 . In consequence of that, exact solutions of both the sub-lattice magnetization $m_{\mathrm{B}}$ and $m_{\mathrm{C}}$ are formally completed.

Finally, we briefly mention an analytical condition determinating a critical behaviour of the considered mixed-spin system. It is quite obvious from the explicit expression of Eqs. (10)-(12) that all sub-lattice magnetization tend necessarily to zero if and only if the sub-lattice magnetization $m_{\mathrm{A}}$ goes to zero. Accordingly, the critical temperature can be located from the condition which is consistent with the known Onsager's exact solution for the critical temperature of the spin- $1 / 2$ Ising model on the rectangular lattice 1

$$
\sinh \left(\beta_{\mathrm{c}} R_{\mathrm{B}} / 2\right) \sinh \left(\beta_{\mathrm{c}} R_{\mathrm{C}} / 2\right)=1,
$$

where $\beta_{\mathrm{c}}=1 /\left(k_{\mathrm{B}} T_{\mathrm{c}}\right)$ is the inverse critical temperature and $T_{\mathrm{c}}$ denotes the critical temperature of the considered model system.

\section{Results and discussion}

Before proceeding to a discussion of the most interesting results it is worth mentioning that the results derived in the preceding section hold regardless of whether ferromagnetic or antiferromagnetic interactions are assumed, irrespective of values of the decorating spins $S_{\mathrm{B}}$ and $S_{\mathrm{C}}$, as well as even if both the single-ion anisotropy parameters $D_{\mathrm{B}}$ and $D_{\mathrm{C}}$ are taken independently of each other. By imposing $S_{\mathrm{B}}=S_{\mathrm{C}}$, $J_{\mathrm{AB}}=J_{\mathrm{AC}}$ and $D_{\mathrm{B}}=D_{\mathrm{C}}$, our results reduce to those acquired for the mixed-spin Ising model on symmetrically decorated square lattices 20 . Therefore, we will restrict ourselves in the present paper only to the particular case with the decorating 


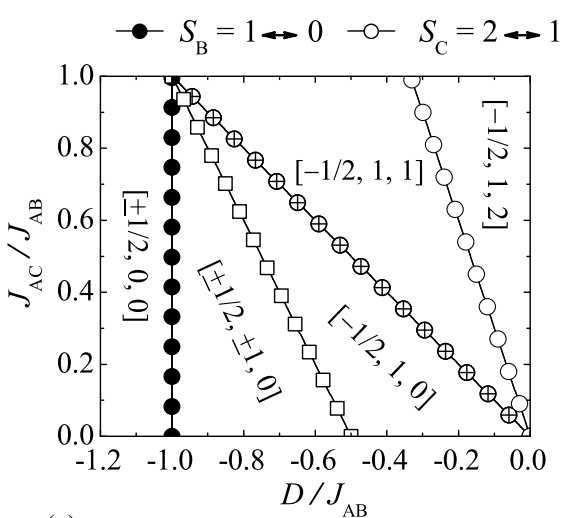

(a)

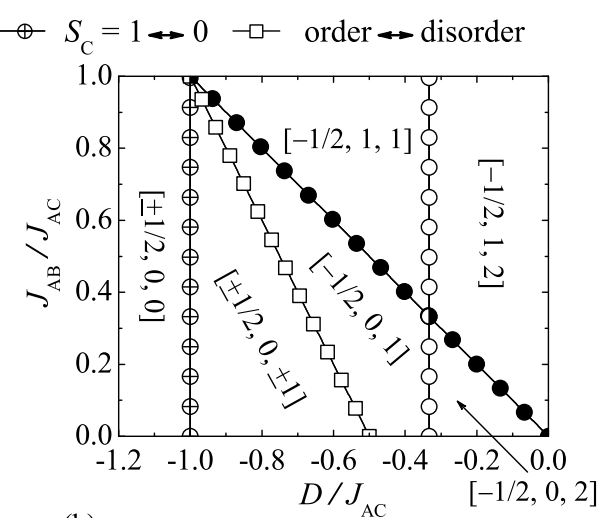

(b)

Fig. 2. Ground-state phase diagrams in the $D-J_{\mathrm{AC}}$ plane for the system with $J_{\mathrm{AB}} \geq J_{\mathrm{AC}}$ [Fig. 2(a)] and in the $D-J_{\mathrm{AB}}$ plane for the system with $J_{\mathrm{AB}} \leq J_{\mathrm{AC}}$ [Fig. 22(b)]. Spin order drawn in square brackets indicates typical spin configurations to emerge within different sectors of the phase diagram. Symbols to be defined in the legend characterize a spin change that occurs at the displayed phase boundaries.

spins of two different integer magnitudes, more specifically, to the case when $S_{\mathrm{B}}=1$ and $S_{\mathrm{C}}=2$. Moreover, in what follows we will restrict both the exchange parameters $J_{\mathrm{AB}}$ and $J_{\mathrm{AC}}$ to positive values. To simplify further discussion, we will also reduce the number of free parameters entering in the Hamiltonian (1) by imposing the same single-ion anisotropy parameter acting on both kinds of decorating spins $\left(D_{\mathrm{B}}=D_{\mathrm{C}}=D\right)$. Other particular cases, in which both the decorating spins are supposed to be half-odd-integer, or integer and half-odd-integer, respectively, will be explored in separate works $36 \mid 37$.

At first, let us take a closer look at a ground-state behaviour of the decorated model. For this purpose, the ground-state phase diagrams are illustrated in Fig. 2 for two particular cases of the anisotropic spin system. Namely, Fig. 2(a) shows the ground-state phase diagram in the $D-J_{\mathrm{AC}}$ plane for the case when the interaction parameter $J_{\mathrm{AB}}$ is stronger than $J_{\mathrm{AC}}$, while Fig. 2(b) demonstrates the ground-state phase diagram in the $D-J_{\mathrm{AB}}$ plane for the case when $J_{\mathrm{AB}}$ is weaker than $J_{\mathrm{AC}}$. In both these figures, the spin order drawn in square brackets indicates a typical spin configuration $\left[S_{\mathrm{A}}, S_{\mathrm{B}}, S_{\mathrm{C}}\right]$ to emerge within the relevant sector of the phase diagram. As one can see, an eventual spin arrangement is unambiguously determined by a mutual competition between the exchange interactions $J_{\mathrm{AB}}$ and $J_{\mathrm{AC}}$ and the single-ion anisotropy $D$. More specifically, the interaction parameters $J_{\mathrm{AB}}$ and $J_{\mathrm{AC}}$ energetically favor the higher spin states of the decorating spin- $S_{\mathrm{B}}$ and spin- $S_{\mathrm{C}}$ atoms, while the easy-plane single-ion anisotropy $(D<0)$ has a tendency to lower their spin states. Accordingly, when the easy-plane single-ion anisotropy reinforces, the system exhibits first-order phase transitions associated with successive lowering 


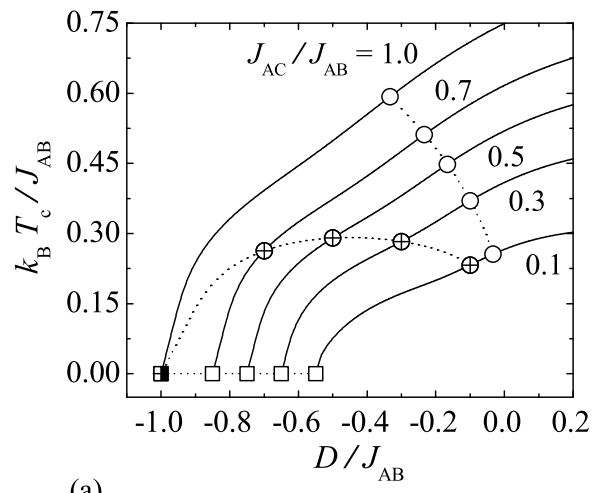

(a)

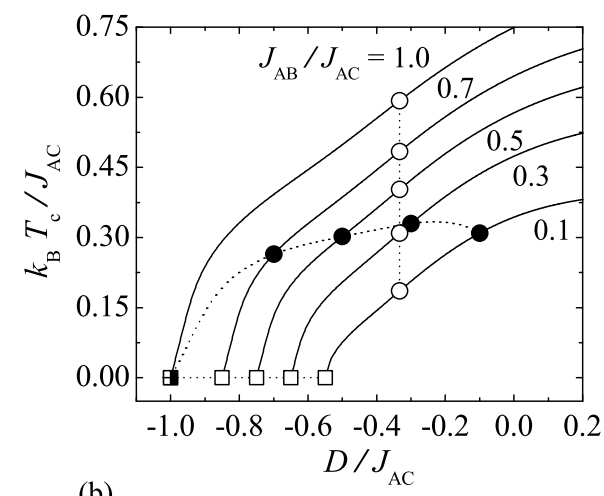

(b)

Fig. 3. Critical temperature as a function of the single-ion anisotropy for several values the ratio $J_{\mathrm{AC}} / J_{\mathrm{AB}}$ in the system with $J_{\mathrm{AB}} \geq J_{\mathrm{AC}}\left[\mathrm{Fig}\right.$. 3(a)] and for several values the ratio $J_{\mathrm{AB}} / J_{\mathrm{AC}}$ in the system with $J_{\mathrm{AB}} \leq J_{\mathrm{AC}}$ [Fig. 3 $\mathrm{b}$ )]. Different symbols characterize the same spin transition as explained in the legend of Fig. 2 Dotted lines, which connect different spin transitions, are guided for eyes only.

of the spin states of decorating atoms. The general condition allocating the groundstate phase transition lines accompanied with the spin change $S_{\mathrm{p}} \leftrightarrow S_{\mathrm{p}}-1$ is given by

$$
\frac{D_{S_{\mathrm{p}}, S_{\mathrm{p}}-1}}{J_{\mathrm{Ap}}}=\frac{1}{1-2 S_{\mathrm{p}}}, \quad \mathrm{p}=\mathrm{B}, \mathrm{C} .
$$

Now, we make some comments on the finite-temperature phase diagrams displayed in Fig. 3. which shows the critical temperature as a function of the single-ion anisotropy for both the investigated cases. All the phase boundaries depicted as solid lines are the unique solutions of the critical condition (14) and in consequence of that, they represent the lines of the second-order phase transitions separating the spontaneously ordered phases (located below the boundary lines) and the disordered paramagnetic phase stable above the relevant phase boundaries.

As it can be seen from a comparison of Figs. 2 and 3 the quite obvious dependence of the critical temperature versus single-ion anisotropy arises for the isotropic case with $J_{\mathrm{AB}}=J_{\mathrm{AC}}$. Indeed, the critical temperature monotonically decreases with decreasing the single-ion anisotropy when $J_{\mathrm{AC}} / J_{\mathrm{AB}}=1.0$ (or equivalently $\left.J_{\mathrm{AB}} / J_{\mathrm{AC}}=1.0\right)$ until the spontaneous order completely vanishes at the boundary value $D_{1,0} / J_{\mathrm{AB}}=-1.0\left(D_{1,0} / J_{\mathrm{AC}}=-1.0\right)$ below which only the disordered phase may exist. This finding is consistent with our expectation, since this boundary single-ion anisotropy is strong enough to force both kinds of decorating spins towards their 'non-magnetic' spin state $S=0$.

However, the situation becomes much more striking when assuming different exchange interactions in the horizontal and vertical directions $\left(J_{\mathrm{AC}} \neq J_{\mathrm{AB}}\right)$. If the 
interaction parameter $J_{\mathrm{AB}}$ is stronger (weaker) $J_{\mathrm{AC}}$, the critical temperature then monotonically decreases with lowering the single-ion anisotropy, but it surprisingly does not tend towards the boundary value $D_{1,0} / J_{\mathrm{AC}}=-1.0\left(D_{1,0} / J_{\mathrm{AB}}=-1.0\right)$ at which all the decorating spin- $S_{\mathrm{C}}\left(\operatorname{spin}-S_{\mathrm{B}}\right)$ atoms tend towards their 'non-magnetic' spin state $S=0$. Actually, one would intuitively expect that all the phases appearing in the parameter region $D<-J_{\mathrm{AC}}\left(D<-J_{\mathrm{AB}}\right)$ should be disordered due to their 'quasi-1D' character. The 2D decorated mixed-spin system indeed behaves as the 'quasi-1D' spin system when $D<-J_{\mathrm{AC}}$ or $D<-J_{\mathrm{AB}}$, since it effectively splits into a set of independent mixed spin- $\left(S_{\mathrm{A}}, S_{\mathrm{B}}\right)$ or spin- $\left(S_{\mathrm{A}}, S_{\mathrm{C}}\right)$ Ising chains depending on whether $J_{\mathrm{AB}}>J_{\mathrm{AC}}$ or $J_{\mathrm{AB}}<J_{\mathrm{AC}}$ is considered, respectively.

On the other hand, one should also bear in mind that the system under investigation is spontaneously long-range ordered if and only if $\sinh \left(\beta R_{\mathrm{B}} / 2\right) \sinh \left(\beta R_{\mathrm{C}} / 2\right)>$ 1 , while it becomes disordered just as $\sinh \left(\beta R_{\mathrm{B}} / 2\right) \sinh \left(\beta R_{\mathrm{C}} / 2\right)<1$ in accordance with the critical condition (14). In the zero-temperature limit, it can be easily verified that

$$
\lim _{T \rightarrow 0}\left[\sinh \left(\beta R_{\mathrm{B}} / 2\right) \sinh \left(\beta R_{\mathrm{C}} / 2\right)\right]=\left\{\begin{array}{ccc}
\infty & \text { if } & D>-J_{\mathrm{AB}} / 2-J_{\mathrm{AC}} / 2 \\
0 & \text { if } & D<-J_{\mathrm{AB}} / 2-J_{\mathrm{AC}} / 2,
\end{array}\right.
$$

what means that the threshold single-ion anisotropy, below which the system becomes disordered at zero as well as non-zero temperatures, is given by the following condition $D_{\mathrm{o}-\mathrm{d}}=-J_{\mathrm{AB}} / 2-J_{\mathrm{AC}} / 2$. This non-trivial phase boundary, which is depicted in the ground-state phase diagram as a hollow-square line (see Fig. 21), cannot be obtained from simple energetic arguments. This result directly proves that all the phases that appear above this order-disorder line are at sufficiently low temperatures spontaneously ordered in spite of their 'quasi-1D' nature. In agreement with the aforementioned arguments, the non-zero critical temperatures to be observed in the parameter space $D<-J_{\mathrm{AC}}\left(D<-J_{\mathrm{AB}}\right)$ manifest the order-disorder transition between the spontaneously ordered and disordered phases even though all the decorating spin- $S_{\mathrm{B}}\left(S_{\mathrm{C}}\right)$ atoms reside in the ground state of the ordered phase the 'non-magnetic' spin state $S=0$ (see Fig. 3). In this respect, the part of second-order phase transition lines starting at $D=-J_{\mathrm{AC}}$ for $J_{\mathrm{AB}}>J_{\mathrm{AC}}$ and at $D=-J_{\mathrm{AB}}$ for $J_{\mathrm{AB}}<J_{\mathrm{AC}}$, and terminating in both the cases at $D=-J_{\mathrm{AB}} / 2-J_{\mathrm{AC}} / 2$ can be identified as the lines of critical points at which the spontaneous long-range order of the 'quasi-1D' spin system disappears.

To provide an independent check of the aforementioned scenario, let us turn our attention to a thermal variation of the total and sub-lattice magnetization. The total magnetization $|m|=\left|m_{\mathrm{A}}+m_{\mathrm{B}}+m_{\mathrm{C}}\right|$ reduced per one site of the original square lattice is plotted against the temperature in Fig. 4 for two investigated particular cases and several values of the single-ion anisotropy. Note that the curves corresponding to the coexistence of two different spin orderings (to emerge in the ground state) are for clarity depicted as dashed lines. Moreover, it is quite obvious from Fig. 4 that the most notable thermal variations of the total magnetization occur in the vicinity of boundary values of the single-ion anisotropy, which are as- 


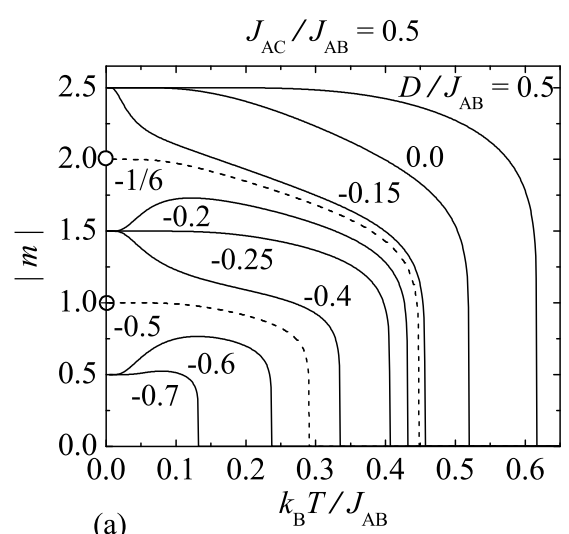

(a)

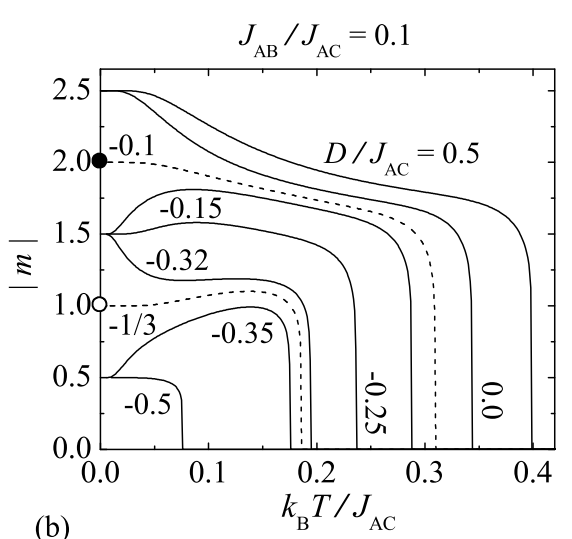

(b)

Fig. 4. Temperature variation of the total magnetization $|m|$ for $J_{\mathrm{AC}} / J_{\mathrm{AB}}=0.5$ [Fig. 4(a)] and $J_{\mathrm{AB}} / J_{\mathrm{AC}}=0.1$ [Fig. 4(b)]. Different symbols characterize the same spin transition as explained in the legend of Fig. 2 Dotted lines, which connect different spin transitions, are guided for eyes only.

sociated with the spin change of the decorating atoms in the ground state. These thermal dependences can be explained with the help of temperature variations of the sub-lattice magnetization. For this purpose, all three sub-lattice magnetization are plotted in Figs. 5 and 6 against the temperature together with the total magnetization. For clear presentation, we choose merely those particular values of the single-ion anisotropy, which are close enough to the single-ion anisotropies at which the spin change of decorating atoms occurs when the same ratio $J_{\mathrm{AC}} / J_{\mathrm{AB}}$ and $J_{\mathrm{AB}} / J_{\mathrm{AC}}$ is fixed as in Fig. 4.

First, let us look more closely on the particular case when $J_{\mathrm{AB}}>J_{\mathrm{AC}}$. According to the thermal variations of the relevant magnetization shown in Fig. 5 (a), one easily observes that slightly above the boundary value $D_{2,1} / J_{\mathrm{AB}}=-1 / 6$, which corresponds in the ground state to the spin change $S_{\mathrm{C}}=2 \leftrightarrow 1$, the total magnetization of the system exhibits a steep initial decrease arising from the thermal excitations $S_{\mathrm{C}}=2 \rightarrow 1$ of the decorating spin- $S_{\mathrm{C}}$ atoms. As a matter of fact, the rapid temperature decrease of the sub-lattice magnetization $m_{\mathrm{C}}$ can be detected. By contrast, the opposite temperature-induced excitations $S_{\mathrm{C}}=1 \rightarrow 2$ are responsible for a rapid increase of the sub-lattice magnetization $m_{\mathrm{C}}$, which in turn causes a gradual increase in the total magnetization when selecting the single-ion anisotropy slightly below the aforementioned boundary value [see Fig. [5(b)]. Similar situation can be found in the vicinity of the boundary value $D_{1,0} / J_{\mathrm{AB}}=-0.5$ associated with the spin change $S_{\mathrm{C}}=1 \leftrightarrow 0$ of the decorating spin- $S_{\mathrm{C}}$ atoms. Actually, the initial decrease in the total magnetization to be detected at $D / J_{\mathrm{AB}}=-0.4$ [see Fig. $5(\mathrm{c})$ ] can be again attributed to rather rapid thermal variation of the sub-lattice magnetization $m_{\mathrm{C}}$, which is thermally more easily disturbed than the sub-lattice magnetization $m_{\mathrm{A}}$ 

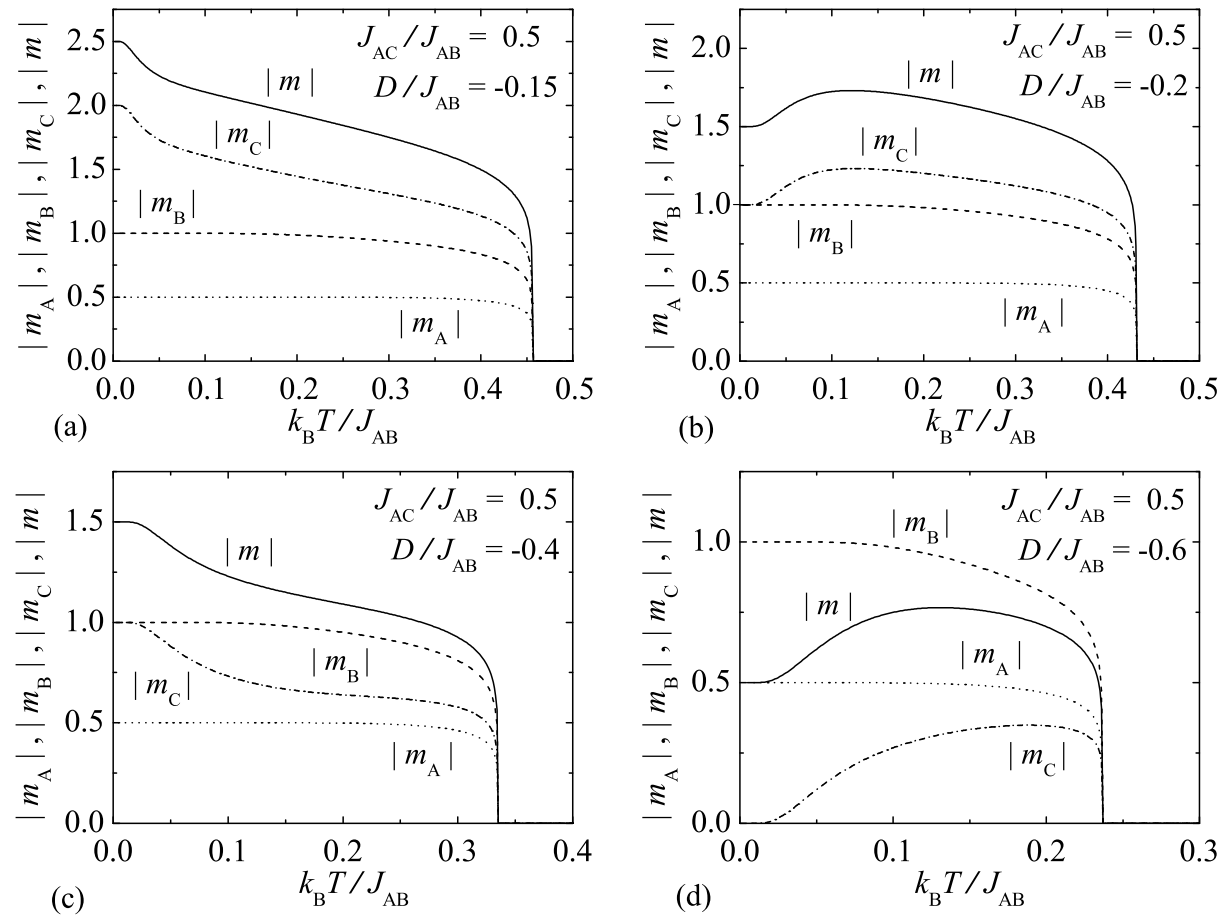

Fig. 5. Temperature variation of the sub-lattice magnetization $\left|m_{\mathrm{A}}\right|$ (dotted lines), $\left|m_{\mathrm{B}}\right|$ (dashed lines), $\left|m_{\mathrm{C}}\right|$ (dashed-dotted lines) and total magnetization $|m|$ (solid lines) for the fixed ratio $J_{\mathrm{AC}} / J_{\mathrm{AB}}=0.5$ and several values of the the single-ion anisotropy parameter $D / J_{\mathrm{AB}}=-0.15$ [Fig. 5(a)], -0.2 [Fig. 5(b)], -0.4 [Fig. 5(c)] and -0.6 [Fig. 5(d)].

and $m_{\mathrm{B}}$ due to the low-lying thermal excitations $S_{\mathrm{C}}=1 \rightarrow 0$. The most interesting temperature dependence of the total magnetization could be, however, expected in the parameter space, where the $2 \mathrm{D}$ decorated mixed-spin system effectively behaves as the 'quasi-1D' system due to its effective splitting into a set of independent mixed spin- $\left(S_{\mathrm{A}}, S_{\mathrm{B}}\right)$ chains. As it can be seen from Fig. $5(\mathrm{~d})$, the thermal variations of sub-lattice magnetization really indicate for $D / J_{\mathrm{AB}}=-0.6$ the 'quasi-1D' character of the spin system, since this value of the single-ion anisotropy is strong enough to force all the decorating spin- $S_{\mathrm{C}}$ atoms towards their 'non-magnetic' state $S=0$ at the zero temperature. According to this, the sub-lattice magnetization $m_{\mathrm{C}}$ increases from zero with the increase of temperature on behalf of the predominating temperature-induced excitations $S_{\mathrm{C}}=0 \rightarrow 1$ of the decorating spin- $S_{\mathrm{C}}$ atoms. Altogether, it might be concluded that a shape of the total magnetization versus temperature dependence is in the mixed-spin system with $J_{\mathrm{AB}}>J_{\mathrm{AC}}$ almost entirely determined by thermal variations of the sub-lattice magnetization $m_{\mathrm{C}}$.

The situation becomes a little bit more involved when assuming the system 
with $J_{\mathrm{AB}}<J_{\mathrm{AC}}$. A detailed analysis of the thermal variations of magnetization reveals that the shape of magnetization curves basically depend on the mutual ratio between the interaction constants $J_{\mathrm{AB}}$ and $J_{\mathrm{AC}}$, more specifically, it depends on whether $J_{\mathrm{AB}} / J_{\mathrm{AC}}>1 / 3$ or $J_{\mathrm{AB}} / J_{\mathrm{AC}}<1 / 3$. In the former case, the temperature curvature of the relevant magnetization detected in the vicinity of boundary singleion anisotropies are very similar to those formerly discussed for the system with $J_{\mathrm{AB}}>J_{\mathrm{AC}}$ and hence, the discussion concerning with their origin is being omitted here for brevity. The only difference consists in the fact that the thermal dependence of sub-lattice magnetization $m_{\mathrm{B}}$ now almost entirely determines the shape of temperature dependence of the total magnetization in contrast to the case with $J_{\mathrm{AB}}>J_{\mathrm{AC}}$, where the sub-lattice magnetization $m_{\mathrm{C}}$ has had a crucial role. However, if one considers $J_{\mathrm{AB}} / J_{\mathrm{AC}}<1 / 3$, the situation becomes even more complicated. The initial increase (decrease) in the total magnetization found at $D / J_{\mathrm{AC}}=0.0(-0.15)$ can be attributed to the thermal excitations $S_{\mathrm{B}}=1 \rightarrow 0\left(S_{\mathrm{B}}=0 \rightarrow 1\right)$ of the spin$S_{\mathrm{B}}$ atoms. This assertion is strongly supported by the rapid temperature decrease (increase) of the sub-lattice magnetization $m_{\mathrm{B}}$ as shown in Fig. 6(a) [Fig. 6(b)]. Moreover, according to the magnetization curves depicted in Fig. 6(b) one easily finds that the value of the single-ion anisotropy $D / J_{\mathrm{AC}}=-0.15$ corresponds to the ground-state region, where all the decorating spin- $S_{\mathrm{B}}$ atoms are driven towards their 'non-magnetic' state $S=0$. In consequence of that, the 2D mixed-spin system should effectively behave as a set of independent mixed spin- $\left(S_{\mathrm{A}}, S_{\mathrm{C}}\right)$ chains (as the 'quasi-1D' system) having the decorating spin- $S_{\mathrm{C}}$ atoms in the 'magnetic' spin state $S=2$. Actually, it is easy to observe from Fig. Fig. 6(b) that the sub-lattice magnetization $m_{\mathrm{B}}$ indeed starts from zero in agreement with our expectations and it is merely the effect of temperature that causes its initial rise. Another notable thermal dependence of the total magnetization can be observed at $D / J_{\mathrm{AC}}=-0.32$, which is slightly above the boundary value $D_{2,1} / J_{\mathrm{AC}}=-1 / 3$ associated with the spin change $S_{\mathrm{C}}=2 \leftrightarrow 1$. As one can see from Fig. 6(c), the total magnetization firstly rapidly decreases and only then slightly increases upon further temperature increase. This interesting non-monotonous behaviour of the total magnetization can be explained as a result of the initial thermal excitations $S_{\mathrm{C}}=2 \rightarrow 1$ of the decorating spin- $S_{\mathrm{C}}$ atoms, while the temperature-induced excitations $S_{\mathrm{B}}=0 \rightarrow 1$ of the decorating spin- $S_{\mathrm{B}}$ atoms overwhelm in the region of higher temperatures. It is indeed quite apparent from Fig. 6)(c) that the sub-lattice magnetization $m_{\mathrm{B}}$ increases much more rapidly in this temperature region than the sub-lattice magnetization $m_{\mathrm{C}}$ declines. This behaviour consequently leads to a slight increase in the total magnetization. Finally, the total magnetization of the system gradually increases with the temperature even if $D / J_{\mathrm{AB}}=-0.35$ is fixed. In this parameter region, the 2D decorated mixed-spin system again effectively behaves as the 'quasi-1D' system due to its effective splitting into a set of independent mixed spin- $\left(S_{\mathrm{A}}, S_{\mathrm{C}}\right)$ chains, but now with the decorating spin- $S_{\mathrm{C}}$ atoms in the 'magnetic' state $S=1$. The observed thermal increase in the total magnetization can be obviously attributed to a rapid thermal variation of the sub-lattice magnetization $m_{\mathrm{B}}$ and $m_{\mathrm{C}}$ arising 

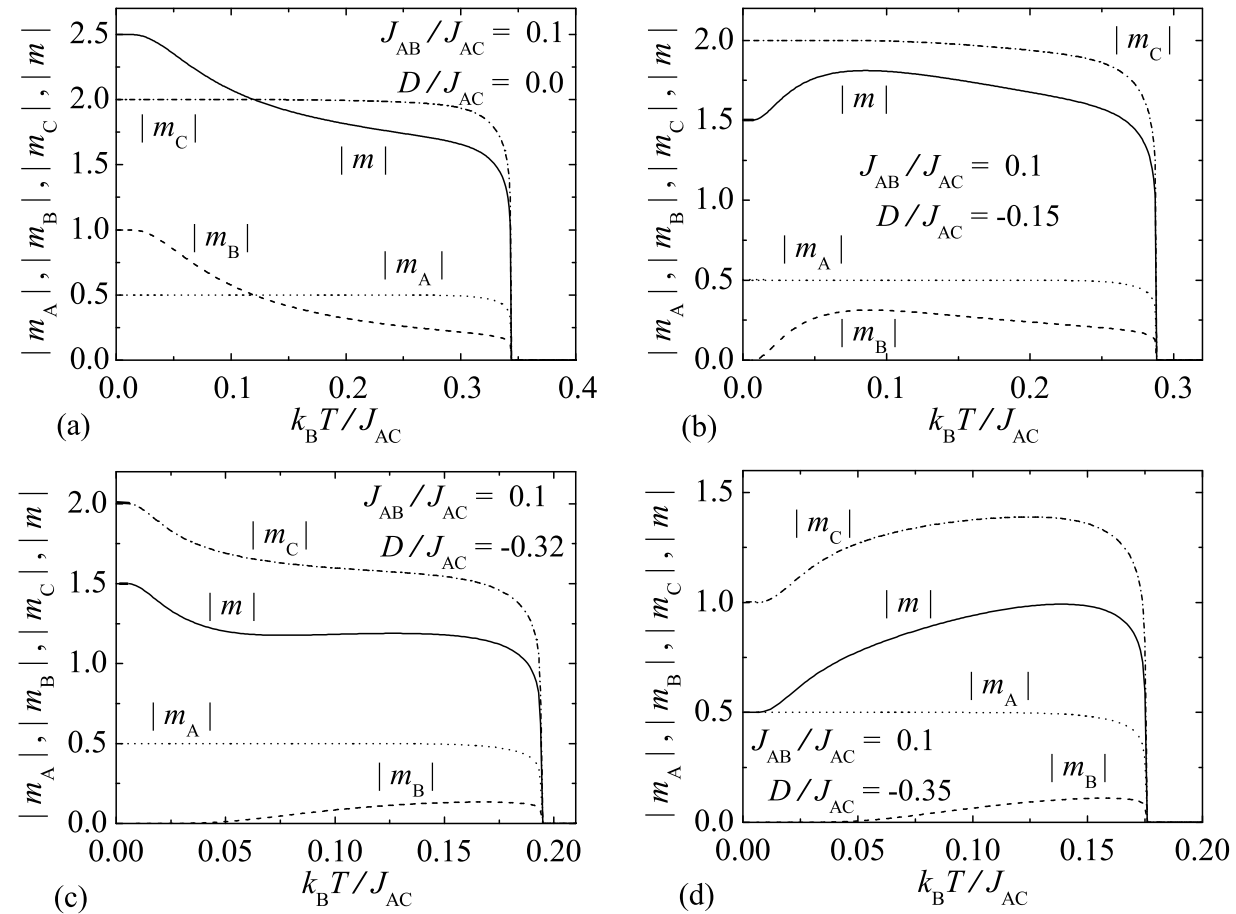

Fig. 6. Temperature variation of the sub-lattice magnetization $\left|m_{\mathrm{A}}\right|$ (dotted lines), $\left|m_{\mathrm{B}}\right|$ (dashed lines), $\left|m_{\mathrm{C}}\right|$ (dashed-dotted lines) and total magnetization $|m|$ (solid lines) for the fixed ratio $J_{\mathrm{AB}} / J_{\mathrm{AC}}=0.1$ and several values of the the single-ion anisotropy parameter $D / J_{\mathrm{AC}}=0.0$ [Fig. 6(a)], -0.15 [Fig. 6(b)], -0.32 [Fig. 6(c)] and -0.35 [Fig. 6(d)].

from the temperature-induced spin excitations $S_{\mathrm{B}}=0 \rightarrow 1$ and $S_{\mathrm{C}}=1 \rightarrow 2$ of the decorating spin- $S_{\mathrm{B}}$ and spin- $S_{\mathrm{C}}$ atoms, respectively.

\section{Conclusion}

In the present article, the mixed-spin Ising model on a decorated square lattice with two different decorating spins of integer magnitudes $S_{\mathrm{B}}$ and $S_{\mathrm{C}}\left(S_{\mathrm{B}} \neq S_{\mathrm{C}}\right)$ placed on horizontal and vertical lattice bonds, respectively, has been investigated within the framework of the generalized decoration-iteration transformation. By the use of this exact mapping procedure, the exact solution for the system under investigation has been attained by establishing a simple mapping relationship with the spin-1/2 Ising model on the corresponding anisotropic square (rectangular) lattice, whose exact solution is known since Onsager's pioneering work 1 . Besides, the aforedescribed mapping transformation method enjoys also immense practical importance, because it is rather general and it implies a possibility of further interesting extensions. 
Actually, this analytical approach can be straightforwardly extended to account also for: (i) the interaction between next-nearest-neighbouring spins $S_{\mathrm{A}}$; (ii) the multi-spin interaction between the decorating spins and their nearest neighbours; (iii) the biaxial single-ion anisotropy acting on the decorating atoms; (iv) other decorated lattices, such as decorated honeycomb or triangular lattices with two or three distinct decorating spins; (v) decorated lattices with two or more decorating spins per one lattice bond; etc. Furthermore, it is also noteworthy that the procedure can be in principle applied to 3D decorated models as well. Nevertheless, we cannot present exact results for any 3D decorated models, since the exact solution of the spin-1/2 Ising model on the corresponding 3D lattice is not known so far.

The most interesting result to emerge from the present study consists in providing an exact evidence for the spontaneous long-range order, which surprisingly appears in the 2D decorated spin system in spite of its 'quasi-1D' character. As a matter of fact, we have found that the $2 \mathrm{D}$ decorated system remains spontaneously ordered even if one sub-lattice of decorating spins (either $S_{\mathrm{B}}$ or $S_{\mathrm{C}}$ ) is forced by a sufficiently strong single-ion anisotropy to be 'non-magnetic' and the system becomes 'quasi-1D' due to the effective splitting into a set of independent mixed spin- $\left(S_{\mathrm{A}}, S_{\mathrm{B}}\right)$ or spin- $\left(S_{\mathrm{A}}, S_{\mathrm{C}}\right)$ chains depending on $J_{\mathrm{AB}}$ is stronger or weaker than $J_{\mathrm{AC}}$, respectively. This finding has an obvious relevance to the understanding of the 'quasi-1D' spin systems inclinable to the spontaneous long-range ordering below some critical temperature, which necessarily do not need to arise from interactions establishing 2D or 3D magnetic structure, but may represent an inherent feature of the 'quasi-1D' system. From this point of view, the exactly solved mixed-spin system presented in this article would be important if some experimental realization of it would confirm the spontaneous ordering notwithstanding of its 'quasi-1D' character.

Although the magnetic structure description of the present mixed-spin Ising model may not be fully realistic for true magnetic materials, it is quite reasonable to expect that the exact solution of this simplified model illustrates many important vestiges of the real critical behaviour. Moreover, the exact solution of theoretical model system may also bring other valuable insights into the thermodynamical properties (magnetization, entropy, specific heat) of true magnetic materials without applying any crude and/or uncontrollable approximative theories.

According to this, the main stimulus for the study of the mixed-spin Ising model on the square lattice with two different decorating spins of magnitudes $S_{\mathrm{B}}$ and $S_{\mathrm{C}}$ placed on horizontal and vertical bonds, respectively, can be viewed in connection with its possible experimental realization. It is therefore of particular interest to mention that polymeric compounds with the architecture of decorated square network have been rather frequently prepared in an attempt to design novel bimetallic coordination compounds. Indeed, the magnetic structure of the decorated square lattice can be found in two numerous series of polymeric coordination compounds with the following general formula: $\left[\mathrm{Ni}(\mathrm{L})_{2}\right]_{2}\left[\mathrm{Fe}(\mathrm{CN})_{6}\right] \mathrm{X}_{2} \mathrm{nH}_{2} \mathrm{O} 38$ and $\mathrm{A}\left[\mathrm{M}_{\mathrm{B}}(\mathrm{L})\right]_{2}\left[\mathrm{M}_{\mathrm{A}}(\mathrm{CN})_{6}\right] \cdot \mathrm{nH}_{2} \mathrm{O}\left(\mathrm{M}_{\mathrm{A}}=\mathrm{Fe}, \mathrm{Mn}, \mathrm{Cr}, \mathrm{Co} ; \mathrm{M}_{\mathrm{B}}=\mathrm{Fe}, \mathrm{Mn}\right) 39$. In the for- 
mer series, the magnetic $\mathrm{Fe}^{3+}\left(S_{\mathrm{A}}=1 / 2\right)$ ions reside the square lattice sites and $\mathrm{Ni}^{2+}\left(S_{\mathrm{B}}=1\right)$ ions decorate each its bond, while in the latter series the low-spin $\mathrm{M}_{\mathrm{A}}{ }^{3+}$ ions such as $\mathrm{Fe}^{3+}\left(S_{\mathrm{A}}=1 / 2\right), \mathrm{Mn}^{3+}\left(S_{\mathrm{A}}=1\right)$, or $\mathrm{Cr}^{3+}\left(S_{\mathrm{A}}=3 / 2\right)$ reside the square lattice sites and the high-spin $\mathrm{M}_{\mathrm{B}}{ }^{3+}$ ions like $\mathrm{Mn}^{3+}\left(S_{\mathrm{B}}=2\right)$ or $\mathrm{Fe}^{3+}$ $\left(S_{\mathrm{B}}=5 / 2\right)$ occupy the decorating lattice sites. Up to now, we are not aware of any trimetallic polymeric compound whose network assembly would consist of two different kinds of magnetic metal ions (decorating spins $S_{\mathrm{B}} \neq S_{\mathrm{C}}$ ) placed on the square net made up by the third magnetic metal ion (by the spin $S_{\mathrm{A}}$ ), but the vast number of the bimetallic coordination compounds from the aforementioned series gives us hope that a targeted synthesis of such trimetallic compounds could be successfully accomplished in the near feature.

\section{Acknowledgements}

The authors would like to express their gratitude to scientific grant agencies for financial support given under the grants VVGS 12/2006, VEGA 1/2009/05 and APVT 20-005204.

1. L. Onsager, Phys. Rev. 65, 117 (1944)

2. R. J. Baxter, Exactly Solved Models in Statistical Mechanics (Academic Press, New York, 1982)

3. J. Oitmaa, J. Phys. A: Math. Gen. 14, 1159 (1981); J. Oitmaa, J. Phys. A: Math. Gen. 15, 573 (1982); P. A. Slotte, J. Phys. C: Solid. St. Phys. 16, 2935 (1983); A. N. Berker and K. Hui, Phys. Rev. B 48, 12393 (1992); K. Minami and M. Suzuki, Physica A 192, 152 (1993)

4. R. Liebmann, Statistical Mechanics of Periodic Frustrated Ising systems, Lecture Notes in Physics (Springer-Verlag, Berlin, 1986), Vol. 251; H. T. Diep and H. Giacomini, in Frustrated Spin Systems, edited by H. T. Diep (Word Scientific, Singapore, 1994); O. Nagai, T. Horiguchi, and S. Miyashita, in Frustrated Spin Systems, edited by H. T. Diep (World Scientific, Singapore, 1994)

5. T. Horiguchi, Phys. Lett. A 113, 425 (1986); F. Y. Wu, Phys. Lett. A 116, 245 (1986); R. Shankar, Phys. Lett. A 117, 365 (1986); V. Urumov, J. Phys. C: Solid. St. Phys. 20, L875 (1987); A. Rosengren and R. Häggkvist, Phys. Rev. Lett. 63, 660 (1989); M. Kolesík and L. Šamaj, Int. J. Mod. Phys. B 3, 1529 (1992); A. Lipowski and M. Suzuki, Physica A 193, 141 (1993); X. D. Mi and Z. R. Yang, Phys. Rev. E 49, 3636 (1994); N. Sh. Izmailian and N. S. Ananikian, Phys. Rev. B 50, 6829 (1994); M. N. Tamashiro and S. R. A. Salinas, Physica A 211, 124 (1994); X. D. Mi and Z. R. Yang, J. Phys. A: Math. Gen. 28, 4883 (1995); T. Horiguchi, Physica A 214, 452 (1995)

6. R. M. F. Houtappel, Physica 16, 425 (1950); H. N. V. Temperley, Proc. Roy. Soc. A 203, 202 (1950); G. H. Wannier, Phys. Rev. 79, 357 (1950); G. F. Newell, Phys. Rev. 79, 876 (1950); K. Husimi and I. Syozi, Progr. Theor. Phys. 5, 177 (1950)

7. I. Syozi, Prog. Theor. Phys. 6, 306 (1951)

8. D. A. Huckaby, J. Phys. C: Solid. St. Phys. 19, 5477 (1986); K. Y. Lin and J. L. Chen, J. Phys. A: Math. Gen. 20, 5695 (1987)

9. T. Utiyama, Prog. theor. Phys. 6, 907 (1951); M. Shinmi and D. A. Huckaby, J. Phys. A: Math. Gen. 20, L465 (1987); K. Y. Lin, C. H. Kao, and T. L. Chen, Phys. 
Lett. A 121, 443 (1987); R. J. Baxter and T. C. Choy, Phys. Lett. A 128, 35 (1988); R. J. Baxter and T. C. Choy, J. Phys. A: Math. Gen. 21, 2143 (1988).

10. J. Strečka, Phys. Lett. A 349, 505 (2005)

11. K. Y. Lin and W. Ma, J. Phys. A: Math. Gen. 16, 3895 (1983)

12. V. G. Vaks, A. I. Larkin, and Yu. N. Ovchinnikov, Zh. Eksp. Teor. Fiz. 49, 1180 (1965) [Sov. Phys. JETP 22, 820 (1966)]; T. Morita, J. Phys. A: Math. Phys. Lett. A 125, 3 (1987); T. C. Choy and R. J. Baxter, J. Phys. A: Math. Phys. Lett. A 125, 365 (1987); T. Chikyu and M. Suzuki, Progr. Theor. Phys. 78, 1242 (1987); R. J. Baxter and T. C. Choy, Proc. R. Soc. London A 423, 279 (1989); K. Y. Lin and F. Y. Wu, J. Phys. A: Math. Gen. 22, 1121 (1989); N. Akutsu and Y. Akutsu, J. Phys. Soc. Jpn. 65, 1233 (1996)

13. V. Urumov, J. Phys. A: Math. Gen. 35, 7317 (2002)

14. C. -F. Sun, X.-M. Kong, and X.-Ch. Yin, Commun. Theor. Phys. 45, 555 (2006)

15. K. Y. Lin and S. C. Wang, Phys. Lett. A 128, 143 (1988); J. Oitmaa and M. Keppert, J. Phys. A: Math. Gen. 35, L219 (2002)

16. M. E. Fisher, Phys. Rev. 113, 969 (1959); K. Yamada, Prog. Theor. Phys. 42, 1106 (1969)

17. L. L. Gonçalves, Phys. Scripta 32, 248 (1985); L. L. Gonçalves, Phys. Scripta 33, 192 (1986); J. W. Tucker, J. Magn. Magn. Mater. 95, 133 (1999); A. Dakhama and N. Benayad, J. Magn. Magn. Mater. 231, 117 (2000); J. Strečka and M. Jaščur, Phys. Rev. B 70, 014404 (2004); M. Jaščur and J. Strečka, Physica A 358, 393 (2005)

18. J. Strečka, Physica A 360, 379 (2006)

19. M. Jaščur and J. Strečka, Condens. Matter Phys. 8, 869 (2005)

20. M. Jaščur, Physica A 252, 217 (1998); A. Dakhama, Physica A 252, 225 (1998); S. Lacková and M. Jaščur, Acta Phys. Slovaca 48, 623 (1998)

21. J. Oitmaa, Phys. Rev. B 72, 224404 (2005)

22. N. R. da Silva and S. R. Salinas, Phys. Rev. B 44, 852 (1991); C. Ekiz and M. Keskin, Physica A 317, 517 (2003); E. Albayrak and M. Keskin, J. Magn. Magn. Mater. 261, 196 (2003); E. Albayrak, Int. J. Mod. Phys. B 17, (2003) 1087; E. Albayrak, Int. J. Mod. Phys. B 18, 3959 (2004); C. Ekiz, J. Magn. Magn. Mater. 293, 759 (2005); C. Ekiz, J. Magn. Magn. Mater. 293, 913 (2005); C. Ekiz, Physica A 347, 353 (2005); C. Ekiz, Physica A 353, 286 (2005); E. Albayrak and A. Alci, Physica A 347, 471 (2005); C. Ekiz and R. Erdem, Phys. Lett. A 352, 291 (2006)

23. A. Lipowski and T. Horiguchi, J. Phys. A: Math. Gen. 28, L261 (1995); J. Strečka, Phys. Stat. Solidi (B) 243, 708 (2006); J. Strečka, L. Čanová, and J. Dely, Phys. Stat. Solidi (B) 243, 1946 (2006)

24. I. Syozi, in Phase Transitions and Critical Phenomena, edited by C. Domb and M. S. Green (Academic Press, New York, 1972), Vol. 1

25. I. Syozi and H. Nakano, Progr. Theor. Phys. 13, 69 (1955); M. Hattori, K. Katsuki, H. Nakano, and I. Syozi, Progr. Theor. Phys. 34, 1050 (1965); M. Hattori, Progr. Theor. Phys. 35, 600 (1966); H. Nakano, Progr. Theor. Phys. 39, 1121 (1968)

26. I. Syozi, Progr. Theor. Phys. 39, 1367 (1968); H. Nakano, Progr. Theor. Phys. 40, 231 (1968); I. Syozi and H. Nakano, Progr. Theor. Phys. 40, 237 (1968); H. Nakano and Y. Yamada, Progr. Theor. Phys. 41, 350 (1969)

27. I. Syozi and S. Miyazima, Progr. Theor. Phys. 36, 1083 (1966); S. Miyazima, Progr. Theor. Phys. 40, 462 (1968)

28. D. A. Huse, M. E. Fisher, and J. M. Yeomans, Phys. Rev. B 23, 180 (1981); M. H. R. Tragtenberg, C. S. O. Yokoi, S. R. Salinas, Rev. Bras. Fis. 18, 161 (1988)

29. I. Syozi, Progr. Theor. Phys. 34, 189 (1965); I. Syozi and S. Miyazima, Progr. Theor. Phys. 36, 1083 (1966); S. Katsura, B. Tsujiyama, in Proceedings of the Conference 
on Critical Phenomena, edited by M. S. Green and J. V. Sergeres (NBS, Washington, 1966), p. 219; A. R. McGrun and M. F. Thorpe, J. Phys. C: Solid St. Phys. 11, 3667 (1978)

30. L. L. Gonçalves, Physica A 110, 339 (1982); T. Horiguchi and L. L. Gonçalves, Physica A 120, 600 (1983); L. L. Gonçalves and T. Horiguchi, Physica A 127, 587 (1984); L. L. Gonçalves and T. Horiguchi, J. Phys. A: Math. Gen. 19, 1449 (1986); R. J. V. dos Santos, S. Countinho, and J. R. L. de Almeida, J. Phys. A: Math. Gen. 19, 3049 (1986)

31. M. E. Fisher, Proc. Roy. Soc. London A 254, 66 (1960); M. E. Fisher, Proc. Roy. Soc. London A 256, 502 (1960); M. Hattori and H. Nakano, Prog. Theor. Phys. 40, 958 (1968); H. Mashiyama and S. Nara, Phys. Rev. B 7, 3119 (1973); W. T. Lu and F. Y. Wu, Phys. Rev. E 71, 046120 (2005); L. Čanová and M. Jaščur, Condens. Matter Phys. 9, 47 (2006)

32. T. Kaneyoshi, J. Phys. Soc. Jpn. 70, 884 (2001); T. Kaneyoshi, Physica A 303, 507 (2002)

33. R. Honmura, T. Kaneyoshi, J. Phys. C 12, 3979 (1979); T. Kaneyoshi, Acta Phys. Pol. A 195, 703 (1993); T. Kaneyoshi, Physica A 229, 166 (1996); T. Kaneyoshi, Physica A 237, 554 (1997)

34. J. H. Barry, M. Khatun, T. Tanaka, Phys. Rev. B 37, 5193 (1988); M. Khatun, J. H. Barry, T. Tanaka, Phys. Rev. B 42, 4398 (1990); J. H. Barry, T. Tanaka, M. Khatun, C. H. Múnera, Phys. Rev. B 44, 2595 (1991); J. H. Barry and M. Khatun, Phys. Rev. B 51, 5840 (1995)

35. R. B. Potts, Phys. Rev. 88, 352 (1952); C. H. Chang, Phys. Rev. 88, 1422 (1952)

36. L. Čanová, J. Strečka, and J. Dely, (unpublished), preprint: cond-mat/0612426

37. J. Strečka, L. Čanová, and M. Jaščur, (unpublished), preprint: cond-mat/0701071

38. M. Ohba, H. Okawa, T. Ito, and A. Ohto, J. Chem. Soc.: Chem. Commun., 1545 (1995); M. Ohba and H. Okawa, Mol. Cryst. Liq. Cryst. 286, 101 (1996); M. Ohba, N. Fukita, H. Okawa, and Y. Hashimoto, J. Am. Chem. Soc. 119, 1011 (1997); H. Z. Kou, W. M. Bu, D. Z. Liao, Z. Jiang, S. P. Yan, Y. G. Fan, and H. G. Wang, J. Chem. Soc.: Dalton Trans., 4161 (1998); M. Ohba and H. Okawa, Coord. Chem. Rev. 198, 313 (2000); H. Okawa and M. Ohba, Bull. Chem. Soc. Jpn. 75, 1191 (2002); N. Usuki, M. Ohba, and H. Okawa, Bull. Chem. Soc. Jpn. 75, 1693 (2002)

39. H. Miyasaka, N. Matsumoto, H. Okawa, N. Re, E. Gallo, and C. Floriani, Angew. Chem. Int. Ed. Eng. 34, 1446 (1995); H. Miyasaka, N. Matsumoto, H. Okawa, N. Re, E. Gallo, and C. Floriani, Angew. Chem. Int. Ed. Eng. 107, 1565 (1995); H. Miyasaka, N. Matsumoto, H. Okawa, N. Re, E. Gallo, and C. Floriani, J. Am. Chem. Soc. 118, 981 (1996); N. Re, E. Gallo, C. Floriani, H. Miyasaka, and N. Matsumoto, Inorg. Chem. 35, 5964 (1996); H. Miyasaka, N. Matsumoto, N. Re, E. Gallo, and C. Floriani, Inorg. Chem. 36, 670 (1997); H. Miyasaka, H. Ieda, N. Matsumoto, N. Re, E. Gallo, and C. Floriani, Inorg. Chem. 37, 255 (1998); N. Re, R. Crescenzi, C. Floriani, H. Miyasaka, and N. Matsumoto, Inorg. Chem. 37, 2717 (1998); M. ClementeLeón, E. Coronado, J. R. Galán-Mascarós, C. J. Goméz-García, T. Wolke, and J. M. Clemente-Juan, Inorg. Chem. 40, 87 (2001); Y. Miyazaki, Q. Wang, Q.-S. Yu, T. Matsumoto, H. Miyasaka, N. Matsumoto, and M. Sorai, Thermochim. Acta 431, $133(2005)$ 